\title{
Parameterized Study of the Test Cover Problem
}

\author{
R. Crowston ${ }^{1}$, G. Gutin ${ }^{1}$, M. Jones ${ }^{1}$, S. Saurabh ${ }^{2}$, and A. Yeo ${ }^{3}$ \\ 1 Royal Holloway, University of London \\ Egham TW20 0EX, UK, \{robert|gutin|markj\}@cs.rhul.ac.uk \\ 2 The Institute of Mathematical Sciences \\ Chennai 600 113, India, saket@imsc.res.in \\ 3 University of Johannesburg \\ Auckland Park, 2006 South Africa, anders.yeo.work@gmail.com
}

\begin{abstract}
In this paper we carry out a systematic study of a natural covering problem, used for identification across several areas, in the realm of parameterized complexity. In the TEST COVER problem we are given a set $[n]=\{1, \ldots, n\}$ of items together with a collection, $\mathcal{T}$, of distinct subsets of these items called tests. We assume that $\mathcal{T}$ is a test cover, i.e., for each pair of items there is a test in $\mathcal{T}$ containing exactly one of these items. The objective is to find a minimum size subcollection of $\mathcal{T}$, which is still a test cover. The generic parameterized version of TEST Cover is denoted by $p(k, n,|\mathcal{T}|)$-TEST Cover. Here, we are given $([n], \mathcal{T})$ and a positive integer parameter $k$ as input and the objective is to decide whether there is a test cover of size at most $p(k, n,|\mathcal{T}|)$. We study four parameterizations for TEST COVER and obtain the following:

(a) $k$-Test Cover, and $(n-k)$-Test Cover are fixed-parameter tractable (FPT), i.e., these problems can be solved by algorithms of runtime $f(k) \cdot \operatorname{poly}(n,|\mathcal{T}|)$, where $f(k)$ is a function of $k$ only.

(b) $(|\mathcal{T}|-k)$-Test Cover and $(\log n+k)$-Test Cover are W[1]-hard. Thus, it is unlikely that these problems are FPT.
\end{abstract}

\section{Introduction}

The input to the Test COVER problem consists of a set of items, $[n]:=$ $\{1,2, \ldots, n\}$, and a collection of distinct sets, $\mathcal{T}=\left\{T_{1}, \ldots, T_{m}\right\}$, called tests. We say that a test $T_{q}$ separates a pair $i, j$ of items if $\left|\{i, j\} \cap T_{q}\right|=1$. Subcollection $\mathcal{T}^{\prime} \subseteq \mathcal{T}$ is a test cover if each pair $i, j$ of distinct items is separated by a test in $\mathcal{T}^{\prime}$. The objective is to find a test cover of minimum size, if one exists. Since it is easy to decide, in polynomial time, whether the collection $\mathcal{T}$ itself is a test cover, henceforth we will assume that $\mathcal{T}$ is a test cover.

TEST COVER arises naturally in the following general setting of identification problems: Given a set of items and a set of binary attributes that may or may not occur in each item, the aim is to find the minimum size subset of attributes (corresponding to a minimum test cover) such that each item can be uniquely identified from the information on which of this subset of attributes it contains. TEST Cover arises in fault analysis, medical diagnostics, pattern recognition, and biological identification (see, e.g., [1112[16]). 
The Test Cover problem has been also studied extensively from an algorithmic view point. The problem is NP-hard, as was shown by Garey and Johnson [7. Moreover, Test Cover is APX-hard [11. There is an $O(\log n)$ approximation algorithm for the problem [16] and there is no $o(\log n)$-approximation algorithm unless $\mathrm{P}=\mathrm{NP}$ [11. These approximation results are obtained using reductions from Test Cover to the well-studied SET COVER problem, where given a collection $\mathcal{S}$ of subsets of $[n]$ covering $[n]$ (i.e., $\cup_{X \in \mathcal{S}} X=[n]$ ) and integer $t$, we are to decide whether there is a subcollection of $\mathcal{S}$ of size $t$ covering $[n]$.

In this paper we carry out a systematic study of TEST COVER in the realm of parameterized complexity 4 . The following will be a generic parameterization of the problem:

$p(k, n, m)$-TEST COVER

Instance: A set $\mathcal{T}$ of $m$ tests on $[n]$ such that $\mathcal{T}$ is a test cover.

Parameter: $k$.

Question: Does $\mathcal{T}$ have a test cover with at most $p(k, n, m)$ tests?

We will consider four parameterizations of Test Cover: $k$-Test Cover, $(m-k)$-Test Cover, $(n-k)$-Test Cover, and $(\log n+k)$-Test Cover. The first parameterization is standard; its complexity is not hard to establish since it is well-known that there is no test cover of size less than $\lceil\log n\rceil$ [1] and the bound is tight. This bound suggests the parameterization $(\log n+k)$-TEST COver (above a tight lower bound). The parameterization $(m-k)$-TEST Cover is a natural parameterization below a tight upper bound. There is always a test cover of size at most $n-1[2]$ and $\mathcal{T}=\{\{1\}, \ldots,\{n-1\}\}$ shows that the bound is tight. Thus, $(n-k)$-TEST COVER is another parameterization below a tight upper bound.

In this paper, we will use some special cases of the following generic parameterization of SET Cover:

$p(k, n, m)$-SET COVER

Instance: A collection $\mathcal{S}$ of $m$ subsets of [ $n]$ covering $[n]$.

Parameter: $k$.

Question: Does $\mathcal{S}$ contain a subcollection of size $p(k, n, m)$ covering $[n]$ ?

Three of our parameterizations for TEST COVER are below or above guaranteed lower or upper bounds. The study of parameterized problems above a guaranteed lower/upper bound was initiated by Mahajan and Raman [14. They showed that some above guarantee versions of MAX CUT and MAX SAT are FPT; in the case of MAX SAT the input is a CNF formula with $m$ clauses together with an integer $k$ (the parameter) and the question is whether there exists an assignment that satisfies at least $m / 2+k$ clauses. Later, Mahajan et al. [15] published a paper with several new results and open problems around parameterizations beyond guaranteed lower and upper bounds. In a breakthrough paper Gutin et

\footnotetext{
${ }^{4}$ Basic notions on parameterized complexity are given in the end of this section.
} 
al. 9] developed a probabilistic approach to problems parameterized above or below tight bounds. Alon et al. 1] combined this approach with a method from Fourier analysis to obtain an FPT algorithm for parameterized MAX $r$-SAT beyond the guaranteed lower bound. In the same paper a quadratic kernel was also given for MAX $r$-SAT. Other significant results in this direction include quadratic kernels for ternary permutation constraint satisfaction problems parameterized above average and results on systems of linear equations over the field of two elements 3 4/10.

We establish parameterized complexity of all four parameterizations of TEST Cover:

(i) Since there is no test cover of size less than $\lceil\log n\rceil, k$-TeST COVER is FPT: if $k<\log n$, the answer for $k$-TEST Cover is No and, otherwise, $n \leq 2^{k}$ and $m \leq 2^{n} \leq 2^{2^{k}}$ and so we can solve $k$-TEST Cover by brute force in time dependent only on $k$.

(ii) In Section 2, we provide a polynomial-time reduction from the INDEPENDENT SET problem to $(m-k)$-TEST COVER to show that $(m-k)$-TeST Cover is W[1]-hard. A reduction from $(m-k)$-Set Cover to $(m-k)$-TeST COver and a result from 8 allows us to conclude that $(m-k)$-TEST Cover is W[1]-complete. Thus, $(m-k)$-TEST Cover is not fixed-parameter tractable unless $\mathrm{FPT}=\mathrm{W}[1]$.

(iii) In Section 3 we prove the main result of this paper: $(n-k)$-TEST Cover is FPT. The proof is quite nontrivial and utilizes a "miniaturized" version of $(n-k)$-Test Cover introduced and studied in Subsection 3.1 .

(iv) Moret and Shapiro [16] obtained a polynomial-time reduction from SET Cover to Test Cover such that the Set Cover problem has a solution of size $k$ if and only if its reduction to TEST COver has a solution of size $k+\lceil\log n\rceil$. Since $k$-SET COVER is W[2]-complete [5], we conclude that $(\log n+k)$-TEST Cover is W[2]-hard. Thus, $(\log n+k)$-Test Cover is not fixed-parameter tractable provided $\mathrm{FPT} \neq \mathrm{W}[2]$.

Basics on Parameterized Complexity. A parameterized problem $\Pi$ can be considered as a set of pairs $(I, k)$, where $I$ is the problem instance and $k$ (usually a nonnegative integer) is the parameter. $\Pi$ is called fixed-parameter tractable if membership of $(I, k)$ in $\Pi$ can be decided by an algorithm of runtime $O\left(f(k)|I|^{c}\right)$, where $|I|$ is the size of $I, f(k)$ is an arbitrary function of the parameter $k$ only, and $c$ is a constant independent from $k$ and $I$. The class of fixed-parameter tractable problems is denoted by FPT.

When the decision time is replaced by the much more powerful $O\left(|I|^{f(k)}\right)$, we obtain the class XP, where each problem is polynomial-time solvable for any fixed value of $k$. There is an infinite number of parameterized complexity classes between FPT and XP (for each integer $t \geq 1$, there is a class $\mathrm{W}[t]$ ) and they form the following tower: $F P T \subseteq W[1] \subseteq W[2] \subseteq \cdots \subseteq W[P] \subseteq X P$. For the definition of classes $\mathrm{W}[t]$, see, e.g., [5] . It is well-known that $\mathrm{FPT} \neq \mathrm{XP}$ and it is widely believed that already $\mathrm{FPT} \neq \mathrm{W}[1]$. Thus, by proving that a problem is $\mathrm{W}[1]$-hard, we essentially rule out that the problem is fixed-parameter tractable 
(subject to FPT $\neq \mathrm{W}[1]$ ). For more information on parameterized complexity, see monographs [5]6]17.

\section{Complexity of $(\boldsymbol{m}-\boldsymbol{k})$-TEST COVER}

In this section we give the hardness result for $(m-k)$-TEST COVER.

Theorem 1. $(m-k)$-TeST Cover is W[1]-complete.

Proof. We will give a reduction from the W[1]-hard $k$-IndePendent Set problem to $(m-k)$-Test Cover. An input to $k$-Independent Set consists of an undirected graph $G=(V, E)$ and a positive integer $k$ (the parameter) and the objective is to decide whether there exists an independent set of size at least $k$ in $G$. A set $I \subseteq V$ is independent if no edge of $G$ has both end-vertices in $I$.

Let $G$ be an input graph to $k$-InDEPEndent SeT with vertices $v_{1}, \ldots, v_{p}$ and edges $e_{1}, \ldots, e_{q}$. We construct an instance of $(m-k)$-TEST Cover as follows. The set of items is $\left\{e_{i}, e_{i}^{\prime}: i \in[q]\right\}$ and the collection of tests is $\left\{T_{j}: j \in[p]\right\} \cup\left\{T_{i}^{\prime}: i \in[q-1]\right\}$, where $T_{j}=\left\{e_{i}: v_{j} \in e_{i}\right\}$, the set of of edges of $G$ incident to $v_{j}$, and $T_{i}^{\prime}=\left\{e_{i}, e_{i}^{\prime}\right\}$.

A set $U$ of vertices of $G=(V, E)$ is a vertex cover if every edge of $G$ has at least one end-vertex in $U$. It is well-known and easy to see that $U$ is a vertex cover if and only if $V \backslash U$ is an independent set. Consider a minimum size vertex cover $U$ of $G$, and a test subcollection $\left\{T_{j}: v_{j} \in U\right\} \cup\left\{T_{i}^{\prime}: i \in[q-1]\right\}$. Observe that the latter is a test cover, since a pair $e_{i}, e_{j}^{\prime}(i \neq j)$ is separated by $T_{\min \{i, j\}}^{\prime}$, as are the pairs $e_{i}, e_{j}$ and $e_{i}^{\prime}, e_{j}^{\prime}$, and a pair $e_{i}, e_{i}^{\prime}$ is separated by $T_{j}$ for some $v_{j} \in U$ such that $v_{j} \in e_{i}$. Such a $v_{j}$ exists since $U$ is a vertex cover.

A test cover must use all $T_{i}^{\prime}$ as otherwise we cannot separate $e_{i}^{\prime}, e_{q}^{\prime}$ for some $i \neq q$. A test cover must also use at least $|U|$ of $T_{j}$ tests. Suppose not, and consider the corresponding set $W$ of vertices, such that $|W|<|U|$. Then every $e_{i}$ is separated from $e_{i}^{\prime}$ by $T_{j}$ for some $v_{j} \in W$, and so $W$ forms a vertex cover, contradicting the minimality of $U$. Hence $G$ has a vertex cover of size $t$ if and only if there is a test cover of size $q-1+t$.

The number of tests is $M=q-1+p$, and so there is a test cover of size $M-k=q-1+p-k$ if and only if $G$ has an independent set with at least $k$ vertices. Since $k$-IndePendent Set is W[1]-hard, $(m-k)$-Test Cover is $\mathrm{W}[1]$-hard as well.

To prove that $(m-k)$-TEST Cover is in W[1], we will use the following reduction of Test Cover to Set Cover by Moret and Shapiro [16. Consider an instance of TEST COVER with set $[n]$ of items and set $\mathcal{T}=\left\{T_{1}, \ldots, T_{m}\right\}$ of tests. The corresponding instance of SET COVER has ground set $V=\{(i, j)$ : $1 \leq i<j \leq n\}$ and set collection $\left\{S_{q}: q \in[m]\right\}$, where $S_{q}=\{(i, j) \in V$ : $T_{q}$ separates $\left.i, j\right\}$. Observe that the instance of TEST CovER has a test cover of size $\mu$ if and only if the corresponding instance of SET COVER has a set cover of size $\mu$. It is proved in [8, Theorem 4] that $(m-k)$-SET Cover is in W[1]. Hence, $(m-k)$-TEST Cover is in W[1] as well. This completes the proof. 


\section{Complexity of $(\boldsymbol{n}-\boldsymbol{k})$-TeSt COVER}

In this section we prove that $(n-k)$-TEST COVER is fixed-parameter tractable. Towards this we first introduce an equivalence relation on $[n]$.

Given a subcollection $\mathcal{T}^{\prime} \subseteq \mathcal{T}$, and two items $i, j \in[n], i \neq j$ we write that $i \equiv \mathcal{T}^{\prime} j$, if $i, j$ is not separated by any tests in $\mathcal{T}^{\prime}$. Clearly, $\equiv_{\mathcal{T}}$ is an equivalence relation on $[n]$. Essentially, each equivalence class is a maximal set $C \subseteq[n]$ such that no pair $i, j \in C$ is separated by a test in $\mathcal{T}^{\prime}$; we say that $C$ is a class induced by $\mathcal{T}^{\prime}$. Observe that $\mathcal{T}^{\prime}$ is a test cover if and only if each class induced by $\mathcal{T}^{\prime}$ is a singleton, i.e., there are exactly $n$ classes induced by $\mathcal{T}^{\prime}$.

\section{$3.1 \quad k$-Mini Test Cover}

To solve $(n-k)$-TEST COVER we first introduce a "miniaturized" version of the problem, namely, the $k$-Mini TEST COVER problem. Here, we are given a set $[n]$ of items and a collection $\mathcal{T}=\left\{T_{1}, \ldots, T_{m}\right\}$ of tests. As with Test Cover, we assume that $\mathcal{T}$ is a test cover. We say that a subcollection $\mathcal{T}^{\prime} \subseteq \mathcal{T}$ is a $k$-mini test cover if $\left|\mathcal{T}^{\prime}\right| \leq 2 k$ and the number of classes induced by $\mathcal{T}^{\prime}$ is at least $\left|\mathcal{T}^{\prime}\right|+k$. We say a test $T$ separates a set $S$ if there exist $i, j \in S$ such that $T$ separates $i, j$. Our main goal in this subsection is to show that the $(n-k)$-Test Cover problem and the $k$-Mini TEST Cover problem are equivalent. Towards this we first show the following lemma.

Lemma 1. Suppose that $\mathcal{T}$ is a test cover for $[n], \mathcal{F} \subseteq \mathcal{T}$ and the number of classes induced by $\mathcal{F}$ is at least $|\mathcal{F}|+k$. Then $\mathcal{F}$ can be extended to a test cover of size at most $n-k$. Moreover, if $\mathcal{T}$ contains all singletons, this is possible by adding only singletons.

Proof. Add tests from $\mathcal{T}$ to $\mathcal{F}$ one by one such that each test increases the number of classes induced by $\mathcal{F}$, until the number of classes is $n$. This can be done, since if we have less than $n$ classes, there is a class $C$ containing at least two items. For $i, j \in C$ there exists a test $T$ in $\mathcal{T} \backslash \mathcal{F}$ that separates $i, j$ which may be added to $\mathcal{F}$. If we are only permitted to add singletons, then pick $T=\{i\}$. Let $\mathcal{F}^{\prime}$ be the subcollection produced from $\mathcal{F}$ in this way. Observe that $\mathcal{F}^{\prime}$ is a test cover. Since $\mathcal{F}$ induces at least $|\mathcal{F}|+k$ classes, we need to add at most $n-(|\mathcal{F}|+k)$ tests to produce $\mathcal{F}^{\prime}$. Thus $\left|\mathcal{F}^{\prime}\right| \leq n-k$, as required.

We now define the notion of a $C$-test as follows.

Definition 1. Let $C \subseteq[n]$. A test $S \in \mathcal{T}$ is a $C$-test if $C \backslash S \neq \emptyset$ and $S \cap C \neq \emptyset$ (i.e. $S$ separates $C$ ). We also define the local portion of a $C$-test $S$ as $L(S)=$ $C \cap S$ and the global portion $G(S)=S \backslash C$.

In order to prove Theorem 2 below we need the following greedy algorithm.

Greedy-mini-test $(\mathcal{T})$ :

Start with $\mathcal{F}=\emptyset$. Add two tests $T_{i}, T_{j}$ from $\mathcal{T}$ to $\mathcal{F}$ if this will increase the number of classes induced by $\mathcal{F}$ by at least 3 . Add a test $T_{i}$ from $\mathcal{T}$ to $\mathcal{F}$ if this will increase the number of classes induced by $\mathcal{F}$ by at least 2 . Stop the construction if we reach $|\mathcal{F}| \geq 2 k-2$. 
Lemma 2. If the algorithm Greedy-mini-test produces a set $\mathcal{F}$ with $|\mathcal{F}| \geq 2 k-2$, then $\mathcal{F}$ is a k-mini test cover.

Proof. Observe that throughout Greedy-mini-test we have at least $\left\lceil\frac{3}{2}|\mathcal{F}|\right\rceil+1$ classes, and when $|\mathcal{F}| \geq 2 k-2$ then $\left\lceil\frac{3}{2}|\mathcal{F}|\right\rceil+1 \geq|\mathcal{F}|+k$. By construction we note that $|\mathcal{F}| \leq 2 k-1<2 k$, which implies that $\mathcal{F}$ is a $k$-mini test cover.

Theorem 2. Suppose that $\mathcal{T}$ is a test cover for $[n]$. Then $\mathcal{T}$ contains a test cover of size at most $n-k$ if and only if $\mathcal{T}$ contains a $k$-mini test cover.

Proof. First suppose that $\mathcal{T}$ contains a $k$-mini test cover $\mathcal{F}$. Then by Lemma 1, $\mathcal{F}$ can be extended to a test cover of size at most $n-k$.

Conversely, suppose $\mathcal{T}$ contains a test cover $\mathcal{F}^{\prime}$ of size at most $n-k$. Now use algorithm Greedy-mini-test on $\mathcal{F}^{\prime}$. If $|\mathcal{F}| \geq 2 k-2$, where $\mathcal{F}$ is produced by Greedy-mini-test, then we are done by Lemma 2, so assume that $|\mathcal{F}|<2 k-2$. This implies that the following holds, as otherwise the algorithm wouldn't have terminated when it did.

1. For every test $T_{i} \in \mathcal{F}^{\prime} \backslash \mathcal{F}, T_{i}$ does not separate more than one class induced by $\mathcal{F}$.

2. For every class $C$ induced by $\mathcal{F}$, and for every pair $T_{i}, T_{j}$ of $C$-tests in $\mathcal{F}^{\prime} \backslash \mathcal{F}$, at least one of $\left(T_{i} \cap T_{j}\right) \cap C,\left(T_{i} \backslash T_{j}\right) \cap C,\left(T_{j} \backslash T_{i}\right) \cap C$ and $C \backslash\left(T_{i} \cup T_{j}\right)$ is empty.

It can be seen that these properties hold even if we add one extra test from $\mathcal{F}^{\prime} \backslash \mathcal{F}$ to $\mathcal{F}$.

Therefore if we add $t$ tests from $\mathcal{F}^{\prime} \backslash \mathcal{F}$, one at a time, this will subdivide a class $C$ into at most $t+1$ classes. Furthermore, since each test separates at most one class, adding $t$ tests from $\mathcal{F}^{\prime} \backslash \mathcal{F}$ to $\mathcal{F}$ will increase the number of classes induced by $\mathcal{F}$ by at most $t$. It follows that $\mathcal{F}^{\prime}$ induces less than $|\mathcal{F}|+k+\left|\mathcal{F}^{\prime} \backslash \mathcal{F}\right|=$ $\left|\mathcal{F}^{\prime}\right|+k \leq n$ classes. But this is a contradiction as $\mathcal{F}^{\prime}$ is a test cover.

By Theorem 2 we get the following result, which allows us to concentrate on $k$-Mini Test Cover in the next subsection.

Corollary 1. The problem $(n-k)$-TEST Cover is FPT if and only if $k$-MINI TEST COVER is FPT.

\subsection{Main Result}

We start with the following easy observation.

Lemma 3. Let $\mathcal{T}$ be a test cover. Let $\mathcal{T}^{*}$ be the test cover formed from $\mathcal{T}$ by adding every singleton not already in $\mathcal{T}$. Then $\mathcal{T}^{*}$ has a $k$-mini test cover if and only if $\mathcal{T}$ also has a $k$-mini test cover.

Proof. Assume $\mathcal{T}^{*}$ has a $k$-mini test cover $\mathcal{F}$. Form $\mathcal{F}^{\prime}$ from $\mathcal{F}$ by removing all singletons. For each singleton removed the number of classes decreases by at most one. Hence, $\mathcal{F}^{\prime}$ induces at least $\left|\mathcal{F}^{\prime}\right|+k$ classes, and $\left|\mathcal{F}^{\prime}\right| \leq 2 k$. Thus, $\mathcal{F}^{\prime}$ is a $k$-mini test cover for $\mathcal{T}$. The other direction is immediate since $\mathcal{T} \subseteq \mathcal{T}^{*}$. 
Due to Lemma 3 hereafter we assume that every singleton belongs to $\mathcal{T}$.

We will apply the algorithm Greedy-mini-test to find a collection $\mathcal{F} \subseteq \mathcal{T}$ of tests. If $|\mathcal{F}| \geq 2 k-2$ then we are done by Lemma 2, so for the rest of the arguments we assume that $|\mathcal{F}|<2 k-2$. By construction, adding any new test to $\mathcal{F}$ increases the number of classes by at most 1 and adding any two new tests to $\mathcal{F}$ increases the number of classes by at most 2. Let the classes created by $\mathcal{F}$ be denoted by $C_{1}, C_{2}, \ldots, C_{l}$. Note that $l \leq 3 k-2$.

Lemma 4. Any test $S \in \mathcal{T} \backslash \mathcal{F}$ cannot be a $C_{i}$-test and a $C_{j}$-test for $i \neq j$.

Proof. For the sake of contradiction, assume such a test $S$ exists. Then adding $S$ to $\mathcal{F}$ will increase the number of classes by at least 2 , a contradiction to the definition of $\mathcal{F}$.

We may assume without loss of generality in the rest of this section that for all $C_{i}$-tests, $S$, we have $\left|S \cap C_{i}\right| \leq\left|C_{i}\right| / 2$. Indeed, suppose $\left|S \cap C_{i}\right|>\left|C_{i}\right| / 2$. Then we may replace $S$ in $\mathcal{T}$ with the test $S^{\prime}=[n] \backslash S$. Observe that two items are separated by $S^{\prime}$ if and only if they are separated by $S$, and so replacing $S$ with $S^{\prime}$ produces an equivalent instance. Furthermore, since $\left|S \cap C_{i}\right|>\left|C_{i}\right| / 2$ we have that $\left|S^{\prime} \cap C_{i}\right| \leq\left|C_{i}\right| / 2$. Note that Lemma 4 still holds after replacing $S$ with $S^{\prime}$, since for all $j \neq i$ either $S^{\prime} \cap C_{j}=\emptyset$ or $C_{j} \subseteq S^{\prime}$.

Lemma 5. Any two $C_{i}$-tests $S, S^{\prime} \in \mathcal{T}$ have either $L(S) \subseteq L\left(S^{\prime}\right)$ or $L\left(S^{\prime}\right) \subseteq$ $L(S)$ or $L(S) \cap L\left(S^{\prime}\right)=\emptyset$.

Proof. For the sake of contradiction, assume $S, S^{\prime}$ do not satisfy this condition. Then $C_{i} \cap\left(S \backslash S^{\prime}\right)$ is non-empty (otherwise, $\left.L(S) \subseteq L\left(S^{\prime}\right)\right)$. Similarly, $C_{i} \cap\left(S^{\prime} \backslash S\right)$ is non-empty. Since $L(S) \cap L\left(S^{\prime}\right) \neq \emptyset, C_{i} \cap S \cap S^{\prime}$ is non-empty. Finally observe that $\left|L(S) \cup L\left(S^{\prime}\right)\right|=|L(S)|+\left|L\left(S^{\prime}\right)\right|-\left|L(S) \cap L\left(S^{\prime}\right)\right| \leq\left|C_{i}\right| / 2+\left|C_{i}\right| / 2-1<\left|C_{i}\right|$. Hence $C_{i} \backslash\left(S \cup S^{\prime}\right)$ is non-empty. Adding $S$ and $S^{\prime}$ to $\mathcal{F}$ divides $C_{i}$ into four classes: $C_{i} \cap\left(S \backslash S^{\prime}\right), C_{i} \cap\left(S^{\prime} \backslash S\right), C_{i} \cap\left(S \cap S^{\prime}\right)$ and $C_{i} \backslash\left(S \cup S^{\prime}\right)$. This contradicts the maximality of $\mathcal{F}$.

An out-tree $T$ is an orientation of a tree which has only one vertex of in-degree zero (called the root); a vertex of $T$ of out-degree zero is a leaf.

We now build an out-tree $O_{i}$ as follows. The root of the tree, $r \in V\left(O_{i}\right)$ corresponds to the set $C_{i}$. Each vertex $v \in V\left(O_{i}\right) \backslash r$ corresponds to a subset, $S_{v} \subseteq C_{i}$ such that there exists a $C_{i}$-test $S \in \mathcal{T}$ with $L(S)=S_{v}$. Note that for a pair of vertices $u, v \in V\left(O_{i}\right)$ if $u \neq v$, then $S_{u} \neq S_{v}$. Add an arc from $v$ to $w$ in $O_{i}$ if $S_{w} \subset S_{v}$ and there is no $u$ in $O_{i}$ with $S_{w} \subset S_{u} \subset S_{v}$. By Lemma 5 we note that $O_{i}$ is indeed an out-tree.

Lemma 6. Every non-leaf in $O_{i}$ has out-degree at least two.

Proof. Let $v$ be a non-leaf in $O_{i}$, and note that $\left|S_{v}\right| \geq 2$. Let $w$ be any child of $v$ in $O_{i}$. By definition there exists an item in $S_{v} \backslash S_{w}$ (as $\left.\left|S_{v}\right|>\left|S_{w}\right|\right)$, say $w^{\prime}$. As there is a singleton $\left\{w^{\prime}\right\} \in \mathcal{T}$ there is a path from $v$ to $w^{\prime}$ in $O_{i}$ and as $w^{\prime} \notin S_{w}$ the path does not use $w$. Therefore $v$ has at least one other out-neighbour. 
We now define the signature of a set $S^{\prime} \subset C_{i}$ as follows.

$$
\operatorname{Sig}\left(S^{\prime}\right)=\left\{G(S): S \in \mathcal{T} \text { and } L(S)=S^{\prime}\right\}
$$

Lemma 7. We have $\left|\left\{\operatorname{Sig}\left(S^{\prime}\right): S^{\prime} \subset C_{i}\right\}\right| \leq 2^{2^{3 k-1}}$.

Proof. Let $\mathcal{S}_{i}$ denote all sets, $S$, with $C_{j} \cap S=\emptyset$ or $C_{j} \subseteq S$ for all $j$ and furthermore $C_{i} \cap S=\emptyset$. Note that $\left|\mathcal{S}_{i}\right| \leq 2^{l-1} \leq 2^{3 k-1}$, since $\left|\left\{C_{1}, \ldots, C_{l}\right\} \backslash\left\{C_{i}\right\}\right| \leq$ $3 k-1$. Note that tests $U$ and $V$ with $L(U)=S^{\prime}=L(V)$ have $G(U) \neq G(V)$, as $U \neq V$. Observe that all $G(S)$ in $\operatorname{Sig}\left(S^{\prime}\right)$ belong to $\mathcal{S}_{i}$ implying that there is at most $2^{\left|\mathcal{S}_{i}\right|}=2^{2^{3 k-1}}$ different choices for a signature.

Lemma 8. There exists a function $f_{1}(k)$ such that either the depth of the tree $O_{i}$ (i.e. the number of arcs in a longest path out of the root) is at most $f_{1}(k)$, or in polynomial time, we can find a vertex $v$ in $O_{i}$ such that if there is a solution to our instance of $(n-k)$-TEST COVER then there is also a solution that does not use any test $S$ with $L(S)=S_{v}$.

Proof. Let $f_{1}(k)=(32 k-1) 2^{2^{3 k-1}}$. Assume that the depth of the tree $O_{i}$ is more than $f_{1}(k)$ and let $p_{0} p_{1} p_{2} \ldots p_{a}$ be a longest path in $O_{i}$ (so $a>f_{1}(k)$ ). By Lemma 7 and by the choice of $f_{1}(k)$, there is a sequence $p_{j_{1}}, p_{j_{2}}, \ldots, p_{j_{32 k}}$, where $1 \leq j_{1}<j_{2}<\cdots<j_{32 k} \leq a$ and all sets corresponding to $p_{j_{1}}, p_{j_{2}}, \ldots, p_{j_{32 k}}$ have the same signature.

Let $S^{*}$ be the set corresponding to $p_{j_{16 k}}$. We will show that if there is a solution to our instance of $(n-k)$-TEST COVER then there is also a solution that does not use any test $S$ with $L(S)=S^{*}$.

Assume that there is a solution to our instance of $(n-k)$-TEST Cover and assume that we pick a solution $\mathcal{T}^{\prime}$ with as few tests, $S$, as possible with $L(S)=S^{*}$. For the sake of contradiction assume that there is at least one test $S^{\prime}$ in our solution with $L\left(S^{\prime}\right)=S^{*}$. By Theorem 2 there is a $k$-mini test cover, $\mathcal{F}^{\prime}$, taken from $\mathcal{T}^{\prime}$. Initially let $\mathcal{F}^{\prime \prime}=\mathcal{F}^{\prime}$. While there exists a vertex $r \in C_{q}$ and $r^{\prime} \in C_{p}(q \neq p)$ which are not separated by $\mathcal{F}^{\prime \prime}$ then add any test from $\mathcal{F}$ which separates $r$ and $r^{\prime}$ to $\mathcal{F}^{\prime \prime}$ (recall that $\mathcal{F}$ is the test collection found by Greedy-mini-test). Note that this increases the size of $\mathcal{F}^{\prime \prime}$ by 1 but also increases the number of classes induced by $\mathcal{F}^{\prime \prime}$ by at least 1 . We continue this process for as long as possible. As $\mathcal{F}^{\prime \prime} \subseteq \mathcal{F} \cup \mathcal{F}^{\prime}$ we note that $\left|\mathcal{F}^{\prime \prime}\right| \leq 2 k+2 k=4 k$. Furthermore, by construction, vertices in different $C_{j}$ 's are separated by tests in $\mathcal{F}^{\prime \prime}$. Also note that the number of classes induced by $\mathcal{F}^{\prime \prime}$ is at least $\left|\mathcal{F}^{\prime \prime}\right|+k$ (as the number of classes induced by $\mathcal{F}^{\prime}$ is at least $\left|\mathcal{F}^{\prime}\right|+k$ ).

For every test, $S$, in $\mathcal{F}^{\prime \prime}$ color the vertex in $O_{i}$ corresponding to $L(S)$ blue. For every vertex, $v \in V\left(O_{i}\right)$, color $v$ red if all paths from $v$ to a leaf in $O_{i}$ use at least one blue vertex and $v$ is not already colored blue. Finally for every vertex, $w \in V\left(O_{i}\right)$, color $w$ orange if all siblings of $w$ (i.e. vertices with the same in-neighbour as $w$ ) are colored blue or red and $w$ is not colored blue or red. We now need the following: 
Claim A: The number of colored vertices in $O_{i}$ is at most $16 k-2$.

Proof of Claim A: As $\left|\mathcal{F}^{\prime \prime}\right| \leq 4 k$ we note that the number of blue vertices is at most $4 k$. We will now show that the number of red vertices is at most $4 k-1$. Consider the forest obtained from $O_{i}$ by only keeping arcs out of red vertices. Note that any tree in this forest has all its leaves colored blue and all its internal vertices colored red. Furthermore, by Lemma 6 the out-degree of any internal vertex is at least 2 . This implies that the number of red vertices in such a tree is less than the number of blue vertices. As this is true for every tree in the forest we conclude that the number of red vertices in $O_{i}$ is less than the number of blue vertices in $O_{i}$ and is therefore bounded by $4 k-1$.

We will now bound the number of orange vertices. Since every orange vertex in $O_{i}$ has at least one sibling colored blue or red (by Lemma 6). and any blue or red vertex can have at most one orange sibling we note that the number of orange vertices cannot be more than the number of vertices colored blue or red. This implies that the number of orange vertices is at most $8 k-1$.

By Lemma 1, we note that some test, $S^{x}$, in $\mathcal{F}^{\prime \prime}$ has $L\left(S^{x}\right)=S^{*}$ (as otherwise extend $\mathcal{F}^{\prime \prime}$ by singletons to a test cover where no test, $S$, in the solution has $L(S)=S^{*}$, a contradiction to our assumption). Now create $\mathcal{F}^{x}$ as follows. Initially let $\mathcal{F}^{x}$ be obtained from $\mathcal{F}^{\prime \prime}$ by removing the test $S^{x}$. Let $p_{j_{i^{\prime}}}$ be an uncolored vertex in $\left\{p_{j_{1}}, p_{j_{2}}, \ldots, p_{j_{16 k-1}}\right\}$ and let $p_{j_{i^{\prime \prime}}}$ be an uncolored vertex in $\left\{p_{j_{16 k+1}}, p_{j_{16 k+2}}, \ldots, p_{j_{32 k-1}}\right\}$ (note that we do not pick $p_{j_{32 k}}$ ). Let $S_{1}^{x}$ be a test in $\mathcal{T}$ with $G\left(S_{1}^{x}\right)=G\left(S^{x}\right)$ and $L\left(S_{1}^{x}\right)$ corresponding to the vertex $p_{j_{i^{\prime}}}$ and let $S_{2}^{x}$ be a test in $\mathcal{T}$ with $G\left(S_{2}^{x}\right)=G\left(S^{x}\right)$ and $L\left(S_{2}^{x}\right)$ corresponding to $p_{j_{i^{\prime \prime}}}$. These tests exist as the signature of all sets corresponding to vertices in $p_{j_{1}}, p_{j_{2}}, \ldots, p_{j_{32 k}}$ are the same. Now add $S_{1}^{x}$ and $S_{2}^{x}$ to $\mathcal{F}^{x}$. The following now holds.

Claim B: The number of classes induced by $\mathcal{F}^{x}$ is at least $\left|\mathcal{F}^{x}\right|+k$.

Proof of Claim B: Let $u, v \in[n]$ be arbitrary. If $u, v \notin C_{i}$ and they are separated by $\mathcal{F}^{\prime \prime}$, then they are also separated by $\mathcal{F}^{x}$, as if they were separated by $S^{x}$ then they will now be separated by $S_{1}^{x}$ (and $S_{2}^{x}$ ). Now assume that $u \in C_{i}$ and $v \notin C_{i}$. If $u \in L\left(S^{x}\right)$ and $u$ and $v$ were separated by $S^{x}$ then they are also separated by $S_{1}^{x}$. If $u \notin L\left(S^{x}\right)$ and $u$ and $v$ were separated by $S^{x}$ then they are also separated by $S_{2}^{x}$. So as $u$ and $v$ were separated by $\mathcal{F}^{\prime \prime}$ we note that they are also separated by $\mathcal{F}^{x}$. We will now show that the number of classes completely within $C_{i}$ using $\mathcal{F}^{x}$ is at least one larger than when using $\mathcal{F}^{\prime \prime}$.

By Lemma 5 we note that deleting $S^{x}$ from $\mathcal{F}^{\prime \prime}$ can decrease the number of classes within $C_{i}$ by at most one (it may decrease the number of classes in $[n]$ by more than one). We first show that adding the test $S_{1}^{x}$ to $\mathcal{F}^{\prime \prime} \backslash\left\{S^{x}\right\}$ increases the number of classes within $C_{i}$ by at least one. As $p_{j_{i^{\prime}}}$ is not colored there is a path from $p_{j_{i^{\prime}}}$ to a leaf, say $u_{1}$, without any blue vertices. Furthermore as $p_{j_{i^{\prime}}}$ is not orange we note that it has a sibling, say $s^{\prime}$, that is not colored and therefore has a path to a leaf, say $u_{2}$, without blue vertices. We now note that $u_{1}$ and $u_{2}$ are not separated in $\mathcal{F}^{\prime \prime}$ (and therefore in $\mathcal{F}^{\prime \prime} \backslash\left\{S^{x}\right\}$ ). However adding the test $S_{1}^{x}$ to $\mathcal{F}^{\prime \prime} \backslash\left\{S^{x}\right\}$ does separate $u_{1}$ and $u_{2}$ (as $u_{1} \in S_{1}^{x}$ but $u_{2} \notin S_{1}^{x}$ ). Therefore the classes within $C_{i}$ has increased by at least one by adding $S_{1}^{x}$ to $\mathcal{F}^{\prime \prime} \backslash\left\{S^{x}\right\}$. 
Analogously we show that adding the test $S_{2}^{x}$ to $\mathcal{F}^{\prime \prime} \cup\left\{S_{1}^{x}\right\} \backslash\left\{S^{x}\right\}$ increases the number of classes within $C_{i}$ by at least one. As $p_{j_{i^{\prime \prime}}}$ is not colored there is a path from $p_{j_{i^{\prime \prime}}}$ to a leaf, say $v_{1}$, without blue vertices. Furthermore as $p_{j_{i^{\prime \prime}}}$ is not orange we note that it has a sibling, say $s^{\prime \prime}$, that is not colored and therefore has a path to a leaf, say $v_{2}$, without blue vertices. We now note that $v_{1}$ and $v_{2}$ are not separated in $\mathcal{F}^{\prime \prime}$ (and therefore in $\mathcal{F}^{\prime \prime} \cup\left\{S_{1}^{x}\right\} \backslash\left\{S^{x}\right\}$, as $p_{j_{i^{\prime}}}$ lies higher in the tree $O_{i}$ and therefore the test $S_{1}^{x}$ does not separate $u$ and $v$ ). However adding the test $S_{2}^{x}$ to $\mathcal{F}^{\prime \prime} \cup\left\{S_{1}^{x}\right\} \backslash\left\{S^{x}\right\}$ does separate $v_{1}$ and $v_{2}$ (as $v_{1} \in S_{2}^{x}$ but $\left.v_{2} \notin S_{2}^{x}\right)$. Therefore the classes within $C_{i}$ has increased by at least one by adding $S_{2}^{x}$ to $\mathcal{F}^{\prime \prime} \cup\left\{S_{1}^{x}\right\} \backslash\left\{S^{x}\right\}$. So we conclude that the number of classes within $C_{i}$ has increased by at least one and as any vertex not in $C_{i}$ is still separated from exactly the same vertices in $\mathcal{F}^{x}$ as it was in $\mathcal{F}^{\prime \prime}$ we have proved Claim B.

By Lemma 1 and Claim B we get a solution with fewer tests, $S$, with $L(S)=$ $S^{*}$, a contradiction.

Suppose the depth of $O_{i}$ is greater than $f_{1}(k)$, and let $S^{*}$ be the set found by the above lemma. Then we can delete all tests, $S$, with $L(S)=S^{*}$ from $\mathcal{T}$ without changing the problem, as if there is a solution for the instance then there is one that does not contain any test $S$ with $L(S)=S^{*}$. Therefore we may assume that the depth of $O_{i}$ is at most $f_{1}(k)$.

Lemma 9. There exist functions $f_{2}(d, k)$ and $f_{3}(d, k)$, such that in polynomial time we can reduce $([n], \mathcal{T}, k)$ to an instance such that the following holds for all vertices $v \in O_{i}$, where $d$ is the length (i.e. number of arcs) of a longest path out of $v$ in $O_{i}$ : (1) $N^{+}(v) \leq f_{2}(d, k)$ and (2) $\left|S_{v}\right| \leq f_{3}(d, k)$.

Proof. Let $v$ be a vertex in $O_{i}$ and let $d$ be the length of a longest path out of $v$ in $O_{i}$. We will prove the lemma by induction on $d$. If $d=0$ then $v$ is a leaf in $O_{i}$ and $N^{+}(v)=0$ and $\left|S_{v}\right|=1$ (as all singletons exist in $\mathcal{T}$ ). So now assume that $d \geq 1$ and the lemma holds for all smaller values of $d$. We note that the way we construct $f_{3}(d, k)$ below implies that it is increasing in $d$.

We will first prove part (1). Let $N^{+}(v)=\left\{w_{1}, w_{2}, w_{3}, \ldots, w_{b}\right\}$ and note that $\left|S_{w_{j}}\right| \leq f_{3}(d-1, k)$ for all $j=1,2, \ldots, b$ (by induction and the fact that $f_{3}(d, k)$ is increasing in $d$ ). Let $Q_{j}$ be the subtree of $O_{i}$ that is rooted at $w_{j}$ for all $j=1,2, \ldots, b$. As part (1) holds for all vertices in $Q_{j}$ we note that there are at most $g(d, k)$ non-isomorphic trees in $\left\{Q_{1}, Q_{2}, \ldots, Q_{b}\right\}$ for some function $g(d, k)$. Furthermore the number of vertices in each $Q_{j}$ is bounded by $2 f_{3}(d-1, k)-1$ by Lemma 6 and induction (using part (2) and the fact that every leaf in $Q_{j}$ corresponds to a singleton in $C_{i}$ and the number of leaves are therefore bounded by $f_{3}(d-1, k)$ ). By Lemma 7 the number of distinct signatures is bounded by $2^{2^{3 k-1}}$. Let $f_{2}(d, k)$ be defined as follows.

$$
f_{2}(d, k)=2 k \cdot g(d, k)\left[2^{2^{3 k-1}}\right]^{2 f_{3}(d-1, k)-1}
$$

So if $b>f_{2}(d, k)$ there exists at least $2 k+1$ trees in $\left\{Q_{1}, Q_{2}, \ldots, Q_{b}\right\}$ which are strongly isomorphic, in the sense that a one-to-one mapping from one to 
the other maintains arcs as well as signatures (a vertex with a given signature is mapped into a vertex with the same signature). Without loss of generality assume that $Q_{1}$ is one of these at least $2 k+1$ trees. We now remove all vertices in $Q_{1}$ as well as all tests $S$ with $L(S)$ corresponding to a vertex in $Q_{1}$. Delete all the items in $S_{w_{1}}$. Let the resulting test collection be denoted by $\mathcal{T}^{\prime}$, and denote the new set of items by $\left[n^{\prime}\right]$. We show this reduction is valid in the following claim.

Claim: This reduction is valid (i.e. $([n], \mathcal{T}, k)$ and $\left(\left[n^{\prime}\right], \mathcal{T}^{\prime}, k\right)$ are equivalent). Proof of Claim: Observe that any $k$-mini test cover in $\mathcal{T}^{\prime}$ is a $k$-mini test cover in $\mathcal{T}$, and so $([n], \mathcal{T}, k)$ is a Yes-instance if $\left(\left[n^{\prime}\right], \mathcal{T}^{\prime}, k\right)$ is a Yes-instance.

For the converse, assume $\mathcal{T}$ contains a $k$-mini test cover $\mathcal{F}^{\prime}$, and for each test $S$ in $\mathcal{F}^{\prime}$, color the vertex in $O_{i}$ corresponding to $L(S)$ blue. We first show we may assume $Q_{1}$ is uncolored. For suppose not, then since $\left|\mathcal{F}^{\prime}\right| \leq 2 k$, then some other tree $Q_{j}$ that is strongly isomorphic to $Q_{1}$ is uncolored. In this case, we may replace the tests $S$ in $\mathcal{F}^{\prime}$ with $L(S)$ corresponding to a vertex in $Q_{1}$, by the equivalent tests $S^{\prime}$ with $L\left(S^{\prime}\right)$ corresponding to a vertex in $Q_{j}$.

So assume $Q_{1}$ is uncolored. Then $\mathcal{F}^{\prime}$ is still a subcollection in $\mathcal{T}^{\prime}$. It remains to show that $\mathcal{F}^{\prime}$ still induces at least $\left|\mathcal{F}^{\prime}\right|+k$ classes over $\left[n^{\prime}\right]$. Observe that this holds unless there is some class $C$ induced by $\left|\mathcal{F}^{\prime}\right|$ that only contains items from $S_{w_{1}}$. But this can only happen if some item in $S_{w_{1}}$ is separated from $S_{w_{j}}$ by a test in $\mathcal{F}^{\prime}$, for all $j \in\{2, \ldots b\}$. But since $b>|\mathcal{F}|+1$, there exists $j \neq 1$ such that $Q_{j}$ is not coloured. Then since $w_{1}, w_{j}$ are siblings, no test in $\mathcal{F}^{\prime}$ can separate $S_{w_{1}}$ from $S_{w_{j}}$. Thus $\mathcal{F}^{\prime}$ induces at least $\left|\mathcal{F}^{\prime}\right|+k$ classes over $\left[n^{\prime}\right]$, and so $\mathcal{F}^{\prime}$ is still a $k$-mini test cover in the new instance. Thus, $\left(\left[n^{\prime}\right], \mathcal{T}^{\prime}, k\right)$ is a YES-instance if and only if $([n], \mathcal{T}, k)$ is a Yes-instance.

By the above claim we may assume that $b \leq f_{2}(d, k)$, which proves part (1). We will now prove part (2). As we have just proved that $b \leq f_{2}(d, k)$ and $\left|S_{w_{j}}\right| \leq f_{3}(d-1, k)$ for all $j=1,2, \ldots, b$, we note that (2) holds with $f_{3}(d, k)=f_{3}(d-1, k) \times f_{2}(d, k)$.

Theorem 3. The $(n-k)$-TEST COVER problem is fixed-parameter tractable.

Proof. Given a test cover, construct a subcollection $\mathcal{F} \subseteq \mathcal{T}$ using algorithm Greedy-mini-test. If $|\mathcal{F}| \geq 2 k$, the instance is a YES-instance since $\mathcal{F}$ induces at least $\frac{3}{2}|\mathcal{F}|$ classes. Otherwise, $|\mathcal{F}|<2 k$ and $\mathcal{F}$ induces at most $3 k$ classes. By Lemma 8, we may assume that $O_{i}$ has depth at most $d=f_{1}(k)$, and by Lemma 9 part (2) we may assume that $\left|C_{i}\right| \leq f_{3}(d, k)$, for each class $C_{i}$ induced by $\mathcal{F}$. Thus $\left|C_{i}\right| \leq f_{3}\left(f_{1}(k), k\right)$.

Hence there are at most $3 k$ classes, the size of each bounded by a function of $k$, so the number of items in the problem is bounded by a function of $k$. Thus, the problem can be solved by an algorithm of runtime depending on $k$ only.

\section{Conclusion}

We have considered four parameterizations of TEST COVER and established their parameterized complexity. The main result is fixed-parameter tractability 
of $(n-k)$-TEST COVER. Whilst it is a positive result, the runtime of the algorithm that we can obtain is not practical and we hope that subsequent improvements of our result can bring down the runtime to a practical level. Ideally, the runtime should be $c^{k}(n+m)^{O(1)}$, but it is not always possible [13.

Acknowledgment This research was partially supported by an International Joint grant of Royal Society.

\section{References}

1. N. Alon, G. Gutin, E.J. Kim, S. Szeider and A. Yeo, Solving Max- $r$-Sat above a tight lower bound. Algorithmica 61 (2011), 638-655.

2. J.A. Bondy, Induced subsets. J. Combin. Th., Ser. B 12 (1972), 201-202.

3. R. Crowston, M. Fellows, G. Gutin, M. Jones, F. Rosamond, S. Thomassé and A. Yeo, Simultaneously satisfying linear equations over $\mathbb{F}_{2}$ : MaxLin2 and Max- $r$-Lin2 parameterized above average. Proc. FSTTCS 2011, LIPICS Vol. 13, 229-240.

4. R. Crowston, G. Gutin, M. Jones, E.J. Kim, and I.Z. Ruzsa, Systems of Linear Equations over $\mathbb{F}_{2}$ and Problems Parameterized above Average. Proc. SWAT 2010, Lect. Notes Comput. Sci. 6139 (2010), 164-175.

5. R. G. Downey and M. R. Fellows. Parameterized Complexity, Springer Verlag, 1999.

6. J. Flum and M. Grohe, Parameterized Complexity Theory, Springer Verlag, 2006.

7. M.R. Garey and D.S. Johnson, Computers and Intractability: A Guide to the Theory of NP-Completeness, Freeman and Co., 1979.

8. G. Gutin, M. Jones and A. Yeo, Kernels for Below-Upper-Bound Parameterizations of the Hitting Set and Directed Dominating Set Problems. Theor. Comput. Sci. 412 (2011), 5744-5751.

9. G. Gutin, E.J. Kim, S. Szeider and A. Yeo, A probabilistic approach to problems parameterized above or below tight bounds. J. Comput. Syst. Sci. 77 (2011), 422429.

10. G. Gutin, L. van Iersel, M. Mnich, and A. Yeo, All Ternary Permutation Constraint Satisfaction Problems Parameterized Above Average Have Kernels with Quadratic Number of Variables. J. Comput. Syst. Sci. 78 (2012), 151-163.

11. B.V. Halldórsson, M.M. Halldórsson, and R. Ravi, On the approximability of the Minimum Test Collection problem. Proc. ESA 2001, Lect. Notes Comput. Sci. 2161 (2001), 158-169.

12. B.V. Halldórsson, J.S. Minden, and R. Ravi. PIER: Proteinidentification by epitope recognition. Proc. Currents in Computational Molecular Biology 2001, 109-110, 2001.

13. D. Lokshtanov, D. Marx, and S. Saurabh, Known Algorithms on Graphs on Bounded Treewidth are Probably Optimal. Proc. SODA 2011, 777-789.

14. M. Mahajan and V. Raman, Parameterizing above guaranteed values: MaxSat and MaxCut. J. Algorithms 31 (1999), 335-354.

15. M. Mahajan, V. Raman, and S. Sikdar, Parameterizing above or below guaranteed values. J. Comput. Syst. Sci. 75 (2009), 137-153.

16. B.M.E. Moret and H.D. Shapiro, On minimizing a set of tests, SIAM J. Scientific \& Statistical Comput. 6 (1985), 983-1003.

17. R. Niedermeier. Invitation to Fixed-Parameter Algorithms, Oxford Univ. Press, 2006. 